\title{
Leber congenital amaurosis
}

INSERM

\section{Source}

INSERM. (1999). Orphanet: an online rare disease and orphan drug data base. Leber congenital amaurosis. ORPHA:65

Leber congenital amaurosis (LCA) is a retinal dystrophy defined by blindness and responses to electrophysiological stimulation (Ganzfeld electroretinogram (ERG)) below threshold, associated with severe visual impairment within the first year of life. 